\title{
Design, Implementation, and Evaluation of an Integrative Pain Management Program in a Primary Care Safety-Net Clinic
}

\author{
Emily Hurstak, MD, MPH, MAS, Maria T. Chao, DrPH, MPA, ${ }^{1,2}$ Kristina Leonoudakis-Watts, $\mathrm{BS}^{3}$ \\ Joseph Pace, $\mathrm{MD}^{3}$ Blue Walcer, $\mathrm{MPH}^{3}$ and Barbara Wismer, MD, $\mathrm{MPH}^{3,4}$
}

\begin{abstract}
Objective: To design, implement, and evaluate a comprehensive Integrative Pain Management Program (IPMP) for patients with chronic pain in a safety-net primary care clinic.

Design: We used a quality improvement "Plan Do Study Act" (PDSA) framework to design, refine, and evaluate an integrative chronic pain program.

Setting: An urban federally qualified health center located in a community with high rates of chronic pain, substance use, and opioid overdose.

Subjects: Eligible participants included individuals with pain for greater than 3 months who were prescribed opioid therapy.

Outcome measures: We designed IPMP using a PDSA framework that promotes continuous evaluation and adaptation of the program to meet the needs of the clinical system. We assessed feasibility and acceptability with program referrals and attendance and evaluated program satisfaction.

Results: The IPMP delivered a 12-week group-based intervention that involved group support, education on pain etiology and treatments, movement-based interventions, mindfulness-based therapies, acupuncture, and massage therapy. One hundred forty-six patients were referred to IPMP; 58 individuals participated in one of the first three cohorts of the program. Sixty-two percent of participants attended at least half of the sessions. Staff and participants reported high levels of satisfaction with IPMP and demand for longitudinal services.

Conclusions: An IPMP delivered within a safety-net primary care clinic could be implemented in a way feasible and acceptable to staff and participants with the support of the local health care system. The application of a PDSA cycle allowed for rigorous implementation and evaluation of a multimodal pain program. Quality improvement frameworks are a strategy to improve and expand the delivery of high-quality patient-centered integrative pain treatments.
\end{abstract}

Keywords: integrative pain management, chronic pain, primary care, safety-net

\section{Introduction}

Background

$\mathbf{P}$ AIN IS ONE OF THE MOST common conditions for which individuals seek medical care. Every year about 100 million Americans report chronic pain symptoms; moreover, pain is the most common cause of disability. ${ }^{1}$ Medications frequently prescribed to treat pain, including nonsteroidal anti-inflammatory medications and opioid analgesics, are associated with nontrivial health risks. ${ }^{2-5}$ Furthermore, medication therapy may not lead to improvements in pain or functional status. 6,7

\footnotetext{
${ }^{1}$ Division of General Internal Medicine, Zuckerberg San Francisco General Hospital and Trauma Center, University of California, San Francisco, San Francisco, California.

${ }^{2}$ Osher Center for Integrative Medicine, University of California, San Francisco, San Francisco, California.

${ }^{3}$ San Francisco Department of Public Health, San Francisco, California.

${ }^{4}$ Department of Family and Community Medicine, University of California, San Francisco, San Francisco, California.
} 
The management of chronic pain is particularly challenging in safety-net clinical settings that serve patients experiencing barriers to medical care due to disparities related to income, ethnicity or race, language, or other factors. Patients receiving care in safety-net settings report high burdens of pain and experience barriers to accessing treatments. ${ }^{1,8,9}$ Consensus guidelines call for improving the availability of multimodal or multidisciplinary pain treatments, defined as programs that combine traditional medical therapy (procedural and/or medications), with nonpharmacologic approaches such as behavioral therapies, physical movement or rehabilitation, and complementary and alternative medicine approaches. ${ }^{10-13}$

San Francisco County has high rates of opioid overdose, with deaths clustering in areas of the city with a concentration of low-income housing. ${ }^{14,15}$ In response to the local opioid overdose epidemic and to clinician frustration with limited treatment options for chronic pain management, a group of patients, stakeholders, clinicians, researchers, and public health leaders collaborated to design and implement the Integrative Pain Management Program (IPMP) at Tom Waddell Urban Health Center (TWUHC).

\section{Setting: TWUHC, San Francisco, CA}

TWUHC provides multidisciplinary services, including primary medical care, mental health care, and substance use treatment, to underserved patients in the Tenderloin and South of Market neighborhoods. Multidisciplinary staff, including physicians and other clinical providers (i.e., nurse practitioners and physician assistants), as well as nurses, pharmacists, medical assistants, behavioral health providers, and substance use counselors, provide comprehensive primary care on-site. Compared to the San Francisco Health Network (SFHN) as a whole, four times as many TWUHC patients were prescribed opioids for pain ( $12 \%$ vs. $3 \%$, respectively). Before the program, TWUHC offered pharmacologic therapy, procedural interventions (e.g., surgery or injections), and physical therapy as standard pain management treatment options.

\section{Methods: Application of a Plan, Do, Study, Act Cycle}

We designed IPMP using a Plan, Do, Study, Act (PDSA) framework that promotes continuous evaluation and adaptation to meet the needs of the clinical system. ${ }^{16} \mathrm{We}$ completed an overall broad-scope PDSA cycle using content from the first three program cohorts. We also completed smaller selfenclosed PDSA cycles to refine each of the first three cohorts. We completed the planning process (Plan) using qualitative feedback from an expert steering committee, focus groups, patient interviews, and an electronic staff survey. We implemented (Do) a multimodal integrative chronic pain program using both group-based delivery of services and one-on-one sessions. We assessed (Study) staff and participant engagement with referrals and attendance data. We evaluated staff and participant satisfaction through focus groups and surveys. We adapted (Act) the IPMP for future cohorts after reviewing and synthesizing the data obtained in PDSA cycles. We collected deidentified data as part of a quality improvement initiative. The University of California San Francisco's Institutional Review Board (IRB) reviewed this protocol and determined it to be exempt.

\section{Methods: Plan-IPMP preparation and development}

IPMP steering committee. TWUHC leadership and local stakeholders, including pain management specialists, SFHN leadership, experts on the local opioid overdose epidemic, behavioral health specialists, and health care providers with expertise in rehabilitation and integrative medicine, formed a multidisciplinary steering committee to discuss program components and implementation. The steering committee met monthly during the planning, implementation, and evaluation stages of the program. During the planning stage, steering committee members reviewed service and educational components of multimodal pain management programs, local expertise on pain management, and examples of multimodal pain programs implemented elsewhere.

Patient and staff focus groups and interviews. The IPMP implementation team conducted focus groups with staff and patients to inform the development of the program. Focus groups with staff focused on determining preference for different program components and to discuss strategies to deliver clinical services, including referrals, eligibility criteria, and clinical communication. Patients discussed their ideal nonpharmacologic pain management strategies. Focus group facilitators prompted patients to offer feedback around the implementation, design, and publicizing of the program.

We also conducted 24 one-on-one interviews and asked potential participants about their goals for pain management, pain treatments they had tried, experiences with group settings, potential barriers to participation, and services that they were interested in trying or felt would be effective. We recorded and transcribed interviews. We identified a set of themes and coded qualitative responses according to repeating consistent themes. A qualitative researcher provided expertise on creating and tallying relevant codes, which were reviewed by the implementation team.

\section{Methods: Do-IPMP design and implementation}

Overall program design. We designed the components of IPMP using a biopsychosocial model of pain, ${ }^{17}$ which considers the biologic, psychologic, and social factors affecting patient function and prognosis. We incorporated integrative medicine components that patients would not have had substantial access to before the program, such as acupuncture, massage, movement, and mindfulness. We chose services considered to be feasible with the greatest potential to benefit participants, taking into account the feedback of experts, staff, patients, and guidelines on chronic pain management. The initial list of services and education topics was developed by the IPMP steering committee relying on expert opinion, guidelines, and what was available through existing staff and funding mechanisms. We did not include cognitive behavioral therapy (CBT) in the program initially because of a lack of available trained staff to provide the service. The implementation team chose a program duration of 12 weeks based on review of models of multimodal pain programs implemented elsewhere.

Patients continued to receive traditional chronic pain treatment throughout their participation in the program. The core 12-week IPMP closed with a graduation celebration. 
Participants received a binder with educational materials and lists of other community resources for pain.

Group-based delivery of services. IPMP involved a weekly 2-h "home group" session, which occurred in a group meeting space on-site. Participants received lunch. Group-based care has been proposed as an effective and efficient way to deliver multimodal pain treatments. ${ }^{18,19}$ Home group sessions started with a "check-in" where participants could share their emotions, thoughts, hopes, and fears related to their health and provide updates since the last session. Group sessions, led by a health educator with years of experience as a group facilitator and a coordinator who had worked as a mental health case manager, involved principles of CBT and fostered peer support. Following the group "check-in," an invited guest (e.g., pharmacist and mindfulness expert) provided information on an educational topic. Topics included "the neurobiology of pain," "medication safety," and "connections between mood and pain."

An ordained lay monk taught mindfulness-based skills that have been shown to be beneficial in chronic pain syndromes. $^{20-22}$ A certified yoga instructor provided education and demonstrated adapted yoga techniques for therapeutic physical movement. The yoga instructor met with a San Francisco Department of Public Health (SFDPH) physical therapist before program to review positional adaptations for different pain syndromes and functional limitations. Participants also received group-based acupuncture utilizing distal points (that do not require full undress) while lying in reclining chairs at weekly home group. In the initial cohort, gardening and nutrition sessions were also offered (Table 1).

One-on-one services. IPMP offered multimodal pain treatments, including acupuncture, movement, mindfulness,

Table 1. Integrative Pain Management Program With Home Group Content

\begin{tabular}{|c|c|c|}
\hline Week & Content & Facilitator(s) \\
\hline 1 & $\begin{array}{l}\text { Orientation-goals, intros, } \\
\text { neuroscience education, } \\
\text { ground rules, self-care }\end{array}$ & $\begin{array}{l}\text { Health educator and } \\
\text { program coordinator } \\
\text { (group facilitators) }\end{array}$ \\
\hline 2 & $\begin{array}{l}\text { Pain stories, successful } \\
\text { treatments, and self-care }\end{array}$ & $\begin{array}{l}\text { Health educator and } \\
\text { program coordinator } \\
\text { (group facilitators) }\end{array}$ \\
\hline 3 & Physical movement & $\begin{array}{l}\text { Certified yoga and } \\
\text { movement instructor }\end{array}$ \\
\hline 4 & Medication education & Clinical pharmacist \\
\hline 5 & Meditation/mindfulness & Mindfulness instructor \\
\hline 6 & $\begin{array}{l}\text { Medication education } \\
\text { (Naloxone training) }\end{array}$ & Clinical pharmacist \\
\hline 7 & Self-massage & $\begin{array}{l}\text { Certified massage } \\
\text { therapist }\end{array}$ \\
\hline 8 & Physical movement & $\begin{array}{l}\text { Certified yoga and } \\
\text { movement instructor }\end{array}$ \\
\hline 9 & Meditation/mindfulness & Mindfulness instructor \\
\hline 10 & Medication education & Clinical pharmacist \\
\hline 11 & Nutrition $^{\mathrm{a}}$ & Nutritionist, educator \\
\hline 12 & $\begin{array}{l}\text { Moving forward, } \\
\text { graduation }\end{array}$ & Program coordinator \\
\hline
\end{tabular}

\footnotetext{
${ }^{a}$ Nutrition was offered during group sessions of the initial cohort only.
}

and massage therapy following home group sessions with an alternating schedule. Participants could also sign up for individual massage therapy and acupuncture sessions during a separate day of the week. IPMP invited each participant to meet one-on-one with a pharmacist to discuss how their medications may work to address a health condition, potential side effects and interactions, and medication safety principles. Finally, IPMP staff assigned each participant a health coach who called them once a week to discuss symptoms, questions, or concerns about the program and agenda setting for the coming week. Health coaches can assist patients to make behavior change and may improve chronic disease management through promotion of self-care. ${ }^{23,24}$ IPMP health coaches were students completing a formal training in health coaching.

Program funding. The program was initially funded through a collaboration between the SFDPH and the Mount Zion Health Fund, a nonprofit entity that supports programs aimed at improving the health of vulnerable populations in San Francisco. The SFDPH provided both direct and in-kind staff support, defined as contribution of staff paid time to the IPMP. SFDPH provided salary support for a full-time program coordinator and in-kind staff support for a health educator, pharmacist, physician acupuncturist, registration staff, and lead physician. The Mount Zion Health Fund provided funds for massage therapy, movement, mindfulness, and acupuncture services. Mount Zion also provided funding to support staff time devoted to evaluation of the initial pilot and for patient participation incentives.

Referral and enrollment of participants. IPMP team members promoted the program by attending clinic meetings, sending e-mails to staff members, and posting promotional materials around the clinic. Eligible patients included primary care patients at TWUHC who had pain for longer than 3 months and who had been prescribed opioid analgesic therapy for pain. IPMP excluded non-English speaking patients, those who were not able to participate in a group setting (as assessed by their primary care provider [PCP]), and pregnant women.

PCPs initiated program referrals through an electronic referral system, but all TWUHC staff members could request a referral for an eligible patient through discussion with the PCP or through an electronic medical record (EMR) message to the PCP. The first three cohorts of IPMP occurred from February through November of 2016. We offered incentives, including grocery gift cards for patients with high levels of participation in home group sessions and those who had participated in each type of service (acupuncture, massage, pharmacy education, and so on) offered by the program. We restricted eligibility for the initial program cohorts to individuals prescribed opioid therapy due to the perception that these participants would benefit most from expanded access to nonpharmacologic pain treatment and because the SFHN registry allowed for easy identification of candidates.

\section{Methods: Study-IPMP evaluation}

As part of a simultaneous PDSA framework evaluation process, IPMP staff collected data on referrals, attendance, and satisfaction with the program. We also enrolled a subset 
of participants in an IRB-approved research study to collect qualitative and quantitative pain and functional status metrics; data will be presented in another publication (submitted for publication).

We assessed baseline (pre-IPMP) staff satisfaction with available pain management treatments and stress related to working with patients with chronic pain, including a singlequestion nonproprietary burnout measure adapted from the Maslach Burnout scale. ${ }^{25,26}$ We repeated a staff survey after the initial three cycles of the program, 9-10 months after initial program implementation. After confirming staff survey data were approximately normally distributed; we calculated summary statistics for five-point Likert Scale responses (mean, median, and standard deviation) and compared pre and postresults using unpaired Student's $t$ tests. We did not have an adequate sample of staff members who completed both pre- and postsurveys to allow paired parametric statistical tests. Staff could elect responses on a five-point Likert Scale (responses ranged not, minimally, moderately, highly, to extremely) on response characteristics such as stressful, frustrating, and confident. We reassigned these categories numerical values ranging from 0 (not characteristic) to 4 (extremely characteristic). Other possible five-point Likert scale responses ranged from very dissatisfied (0), dissatisfied (1), neutral (2), satisfied (3), and very satisfied (4) and no access (0), limited access (1), some access (2), good access (3), and abundant access (4). The single question burnout scale is graded on a scale of $1-5$, where a higher score is consistent with greater perceived degree of burnout.
We administered a participant survey at program graduation. We asked participants a series of statements measured on a five-point Likert Scale $(1=$ strongly disagree to $5=$ strongly agree), including "If the pain program was offered again, I would be interested in coming" and "I would recommend the pain program to other people with pain." Participants also rated their level of satisfaction with specific aspects of IPMP (e.g., acupuncture, interactions with the health coach, and overall experience).

\section{Results}

\section{Findings of the plan and do phases}

We identified several controversial implementation decisions during the planning and design phase. In focus groups and informal communication, staff voiced concerns about the proposed program eligibility restriction to patients who were prescribed opioid therapy. Staff felt that patients who had recently undergone discontinuation of opioids or those who were ineligible for opioid therapy might be ideal patient participants in the program. Key themes of staff focus groups are summarized in Table 2.

Patients voiced concerns that some might view participation in the program as an alternative to opioid therapy or as a threat to ongoing opioid therapy. They recommended that descriptions of the program promote IPMP as an additional patient resource to help with pain management. Table 1 summarizes patient focus group and interview themes.

During the planning phase, the team decided NOT to make program participation mandatory for patients receiving chronic

Table 2. Staff and Patient Qualitative Themes on Developing an Integrative Pain Management Program

\section{Referrals and scheduling}

Use existing electronic referral system to facilitate referrals and allow preprogram communication between clinicians and program staff.

Allow any staff member to refer to reduce barriers to referral.

Minimize delays between referral and program start.

Try to schedule program during afternoons.

\section{Marketing the program}

Provide clinical staff with information about program components through promotional materials so they can publicize program to patients.

Ensure patients understand that they can receive usual treatment from their doctor regardless of program participation.

Use simple and common language, that is, don't call mindfulness "meditation," demonstrate what an acupuncture treatment looks like, etc.

\section{Program components}

Expanded access to nonpharmacologic treatments, including acupuncture, behavioral therapy, electrical stimulation (TENS or other), exercise or movement-based therapies, massage, nutrition education, spiritual or mindfulness component, water-based therapy.

Education on medication safety and overdose prevention with naloxone.

\section{Program design and delivery}

Minimize individual visits (i.e., schedule group sessions).

Keep the group small and enforce privacy guidelines

Keep the tone of discussion positive

Document encounters in the electronic medical record.

Communicate with PCPs through EMR messaging.

Offer longitudinal services to program graduates.

Offer food. Provide a comfortable space for group meetings and services.

\section{Barriers to participation}

Accessibility issues: distance from home, mobility struggles, getting to appointments.

Timing of appointments, too many appointments.

Fear of group settings (judgment of others, confidentiality, etc.)

Headings bolded for emphasis.

EMR, electronic medical record; PCP, primary care provider. 
opioid therapy while acknowledging that a policy like this would ensure attendance. Furthermore, program staff decided NOT to make home group attendance a requirement for participation in popular one-on-one services like acupuncture and massage. IPMP instead offered these popular services directly after the home group to promote attendance at group sessions.

\section{Findings of the study phase}

Feasibility and acceptability of program. Staff engagement: One hundred percent of primary care providers at the clinic $(n=15)$ referred at least one patient. Patient engagement: Of 146 total referrals, 19 were ineligible, 25 were unreachable, 23 were unconfirmed or undecided about participation, and 6 declined to participate; 58 out of the remaining 73 patients (79\%) enrolled in IPMP and attended at least one home group session (mean attendance $=6.4$ sessions). We found moderately high patient participation: $62 \%$ attended at least 6 out of 12 home group sessions. Similar to the overall demographics of TWUHC patients, participants were low income and were racially/ethnically diverse.

Staff satisfaction. Thirty-one staff members completed the pre-IPMP survey, including PCPs, nurses, medical as- sistants, social workers, and clerical staff. Thirty-three staff members completed a postprogram survey (Table 3 ).

Before IPMP, staff described working with patients who have chronic pain as "moderately to very stressful (2-3)" and "moderately frustrating (2)." Staff reported that they were "neutral (2)" or "dissatisfied (1)" with available options for chronic pain (mean 1.5, standard deviation $[\mathrm{SD}]=0.9$ ). Staff members rated their level of burnout with a mean score of $2.7(\mathrm{SD}=1.0)$ where a score of 2 is consistent with "occasionally I am under stress, and I don't always have as much energy as I once did, but I don't feel burned out'" and a score of 3 is consistent with "I am definitely burning out and have one or more symptoms of burnout, such as physical or emotional exhaustion." Staff reported limited access to multimodal treatments (range of responses: $1=$ limited access, $2=$ some access), but were only minimally (1) to moderately (2) confident that IPMP would improve patient function and reduce opioid risk (Table 3).

In a staff survey administered after completion of the first three cohorts of the program, we observed a statistically significant improvement in satisfaction with available options for pain treatment $(1.5$ vs. $2.7, p<0.001 ; 1=$ dissatisfied, $2=$ neutral, $3=$ satisfied). Staff reported that they were "satisfied"

Table 3. Pre- Versus Postintegrative Pain Management Program Surveys with Primary Care Clinic Staff

\begin{tabular}{|c|c|c|c|c|c|}
\hline & \multicolumn{2}{|c|}{ Pre-IPMP $(\mathrm{n}=31)$} & \multicolumn{2}{|c|}{ Post-IPMP $(\mathrm{n}=33)$} & \multirow[b]{2}{*}{$\mathrm{p}$} \\
\hline & Mean & $S D$ & Mean & $S D$ & \\
\hline Stress when working with patients with chronic pain ${ }^{\mathrm{a}}$ & 2.30 & 0.90 & 2.00 & 1.14 & 0.25 \\
\hline Frustration when working with patients with chronic pain ${ }^{a}$ & 2.20 & 1.00 & 1.72 & 1.02 & 0.06 \\
\hline $\begin{array}{l}\text { Satisfaction with treatment options available } \\
\text { for patients with chronic pain }\end{array}$ & 1.50 & 0.90 & 2.70 & 0.98 & 0.0001 \\
\hline Overall burnout ${ }^{\mathrm{b}}$ & 2.70 & 1.00 & 2.28 & 0.96 & 0.10 \\
\hline \multicolumn{6}{|l|}{ Level of confidence in IPMP } \\
\hline Staff confidence to engage patients & 1.90 & 0.80 & 2.34 & 1.04 & 0.07 \\
\hline IPMP to improve function & 1.90 & 0.70 & 2.44 & 0.70 & 0.005 \\
\hline IPMP to reduce opioid risk & 1.60 & 0.60 & 2.44 & 0.77 & 0.0001 \\
\hline IPMP to minimize opioids in treatment ${ }^{\mathrm{c}}$ & - & & 2.29 & 1.01 & \\
\hline \multicolumn{6}{|l|}{ Access to treatment options $\mathrm{s}^{\mathrm{a}, \mathrm{d}}$} \\
\hline Acupuncture & 1.10 & 0.90 & - & & \\
\hline Massage & 1.10 & 0.90 & - & & \\
\hline Medication education & 1.80 & 1.10 & - & & \\
\hline Mindfulness & 1.30 & 1.10 & - & & \\
\hline Nutrition & 1.20 & 1.00 & - & & \\
\hline Physical movement & 1.40 & 0.90 & - & & \\
\hline \multicolumn{6}{|l|}{ Is treatment helpful for patient ${ }^{\mathrm{a}, \mathrm{d}}$} \\
\hline Acupuncture & 3.20 & 0.80 & - & & \\
\hline Massage & 3.10 & 1.00 & - & & \\
\hline Medication education & 2.90 & 0.70 & - & & \\
\hline Mindfulness & 3.40 & 0.70 & - & & \\
\hline Nutrition & 2.70 & 1.00 & - & & \\
\hline Physical movement & 3.00 & 0.70 & - & & \\
\hline \multicolumn{6}{|l|}{ Satisfaction with IPMP } \\
\hline Overall satisfaction with program & - & & 3.06 & 1.11 & \\
\hline Patient experience with program & - & & 3.10 & 0.76 & \\
\hline
\end{tabular}

Bolded values achieve statistical significance at a $p<0.05$ level.

$p$ Values based on unpaired t tests of pre- versus post-IPMP surveys. Survey repeated after initial three program cohorts.

${ }^{a}$ Based on a five-point likert scale with possible range from 0 to 4.

${ }^{\mathrm{b}}$ Based on a single question burnout scale adapted from Maslach Inventory with a possible range of responses from 1 to 5.

'Only asked in postsurvey.

${ }^{\mathrm{d}}$ Post-IPMP data not reported due to low response rate.

IPMP, Integrative Pain Management Program; SD, standard deviation. 
with IPMP, mean $3.1(\mathrm{SD}=1.11)$, and rated patient experiences with IPMP as "satisfied," mean $3.1(\mathrm{SD}=0.76)$. Staff described IPMP as "effective (3)" in improving functioning of patients, mean $2.9(\mathrm{SD}=0.75)$. Staff responses on the effectiveness of IPMP in reducing opioid misuse or minimizing opioids in treatment ranged from neutral (2) to effective (3): mean scores $2.4(\mathrm{SD}=0.77)$ and $2.4(\mathrm{SD}=1.01)$, respectively. We observed statistically significant increases in staff confidence in IPMP's ability to improve functioning $(p=0.005)$ and reduce opioid risk $(p<0.001)$. We did not find statistically significant changes in staff reported stress, frustration, or burnout level related to caring for patients with chronic pain.

Participant satisfaction. We measured overall high acceptability of IPMP (Table 4). All participants agreed that they would recommend the pain program to others. A majority of participants agreed that the pain program was a safe place to discuss difficult issues (97\%) and was supportive emotionally (92\%). About two thirds (64\%) felt that they were better able to manage chronic pain on their own. Nearly all participants $(97 \%)$ reported being satisfied or completely satisfied with their experience with IPMP.

Findings of the act phase: IPMP evolution and expansion

In response to feedback from patients and staff, IPMP team leadership modified program eligibility, design, and components throughout the PDSA cycles. The IPMP is now open to all patients with chronic pain at TWUHC and three other primary care clinics in the SFHN. The eligibility criteria expanded to include patients with chronic pain not on opioid therapy. The expansion in eligibility was prompted by both clinician and patient desire for broader participation in the program's services. The initial pilot eligibility criteria were considered too narrow given the prevalence of chronic pain and limited treatment options within the network. To improve participant exposure and participation, we integrated the mindfulness and movement components into the home group to encourage more individuals to try these services. The home group now involves a CBT curriculum with a trained behavioral health clinician. CBT had been a desired component of the pilot program that we were unable to fulfill due to initial staffing limitations. With program success and advocacy of leadership, this service is now available. The program was temporarily expanded to a 16week duration, but then subsequently shortened after accumulating additional staff and participant feedback. There were a range of opinions on the appropriate duration of this type of intervention, but with the creation of a graduate group, many of these concerns were addressed.

IPMP participants may sign-up for a weekly graduate's group that offers peer support, education on topics related to chronic pain, practice of mindfulness, and movementbased therapies, including qigong, as well as massage and

Table 4. Participant Satisfaction with the Integrative Pain Management Program $(N=36)$

\begin{tabular}{lc}
\hline & $\begin{array}{c}\text { Agree or strongly } \\
\text { agree }\end{array}$ \\
\hline The program helped me learn how to reduce my pain. & $83(30)$ \\
I use what I learned in the pain program in my daily life. & $86(31)$ \\
The program helped improve my day-to-day functioning. & $81(29)$ \\
The program helped improve my quality of life. & $72(26)$ \\
As a result of the program, I am better able to manage my chronic pain on my own. & $64(23)$ \\
If the pain program was offered again, I would be interested in coming. & $94(34)$ \\
It is likely that I would come for additional services offered in the pain program. & $89(32)$ \\
I would recommend the pain program to other people with pain. & $100(36)$ \\
The clinic where the pain program was offered was convenient for me. & $86(31)$ \\
The pain program was supportive to me emotionally. & $92(33)$ \\
The other members of the group treated me with respect. & $100(36)$ \\
The leaders of the group treated me with respect. & $100(36)$ \\
The pain program was a safe place to discuss difficult issues. & $97(35)$ \\
\hline
\end{tabular}

Aspects of the pain program

Home groups

Acupuncture 1-on-1

Acupuncture group visit

Massage

Movement

Mindfulness

Pharmacist visit

Interactions with health coach

Completing surveys and interviews

Scheduling pain program services

Interactions with the providers and clinicians in the pain program

Reminder calls

Overall experience with the pain program
Satisfied or completely satisfied \% (n)

$94(34)$

$87(20)$

$91(21)$

97 (29)

$97(30)$

$100(28)$

$92(23)$

$83(25)$

$94(34)$

$94(30)$

97 (33)

$94(32)$

$97(34)$

\footnotetext{
aVariable total, $N(N=23-35$; some individuals responded "not applicable" to questions). Survey administered at program graduation
} after 12 weeks. 
acupuncture sessions. The success of the initial pilot program engendered support for ongoing financial support of the program. The SFDPH now provides funds for program services, including massage, acupuncture, movement, and mindfulness, through a practice improvement program. Other staff members continue to be supported by "in-kind" salary support. While this funding is not guaranteed beyond an annual basis, there is broad support to continue to support this initiative within San Francisco's safety-net health care system.

\section{Discussion}

There are few published studies describing multimodal pain programs delivered in primary care settings, particularly in safety-net settings. ${ }^{19,27,28}$ This is the only program of its kind in the San Francisco Bay Area, but there are other similar models that have been implemented in Northern California, Los Angeles, Oregon, and Massachusetts. ${ }^{29}$ Implementation of a PDSA cycle for the initial three cohorts of the IPMP allowed us to refine the program to achieve satisfaction and promote sustainability. We are not aware of other publications describing implementation of a PDSA cycle to evaluate a multimodal pain program.

Initial patient and staff input increased the likelihood of program success by refining the program design and ensuring participant and staff buy-in. IPMP staff presented the evaluation measures at periodic meetings with key stakeholders, including TWUHC staff and the IPMP steering committee. Participants, staff, and the steering committee provided feedback around interpretation of results and next steps to improve the program. IPMP sought additional feedback from staff and patient participants through repeated focus groups, participant surveys, and monthly staff meetings. Despite requiring significant time commitments from staff and patients, we observed high rates of participation in the program and evaluation processes.

We found that a multimodal integrative pain program implemented in a safety-net clinical setting was feasible and acceptable to patients and staff and produced high rates of participation and satisfaction. Using a PDSA cycle to design, implement, and evaluate an IPMP fostered the creation of a sustainable and effective program that continues to evolve. A PDSA framework allows for the timely identification of barriers and challenges and provides ongoing monitoring of program to promote high quality care. PDSAdirected evaluations are a strategy for the rigorous implementation and evaluation of integrative medicine models, which may promote efficient and patient-centered system improvement and innovation.

\section{Acknowledgments}

We acknowledge the participants of the IPMP steering committee, TWUHC staff, and patient participants for their involvement in program development, implementation, and evaluation. Jimmy $\mathrm{He}$ provided assistance coordinating and conducting interviews and collecting attendance and quality improvement data. Frank Sidders provided ongoing support on program monitoring, including referrals, attendance, and satisfaction. Funding for the study was provided through the Mount Zion Health Fund. The funding source had no role or involvement in the design and conduct of the study.

\section{Author Disclosure Statement}

No competing financial interests exist.

\section{References}

1. Institute of Medicine Committee on Advancing Pain Research Care and Education. Relieving Pain in America: A Blueprint for Transforming Prevention, Care, Education, and Research. Washington, DC: National Academies Press (US) National Academy of Sciences.; 2011.

2. Calcaterra S, Glanz J, Binswanger IA. National trends in pharmaceutical opioid related overdose deaths compared to other substance related overdose deaths: 1999-2009. Drug Alcohol Depend 2013;131:263-270.

3. Lewis JD, Kimmel SE, Localio AR, et al. Risk of serious upper gastrointestinal toxicity with over-the-counter nonaspirin nonsteroidal anti-inflammatory drugs. Gastroenterology 2005;129:1865-1874.

4. Schneider V, Levesque LE, Zhang B, et al. Association of selective and conventional nonsteroidal antiinflammatory drugs with acute renal failure: A population-based, nested case-control analysis. Am J Epidemiol 2006;164:881889.

5. Chen LH, Hedegaard H, Warner M. Drug-poisoning deaths involving opioid analgesics: United States, 1999-2011. NCHS Data Brief 2014:1-8.

6. Noble M, Treadwell JR, Tregear SJ, et al. Long-term opioid management for chronic noncancer pain. Cochrane Database Syst Rev 2010:CD006605.

7. Chou R, Deyo R, Friedly J, et al. Systemic pharmacologic therapies for low back pain: A systematic review for an American College of Physicians Clinical Practice Guideline. Ann Intern Med 2017;166:480-492.

8. Knight KR, Kushel M, Chang JS, et al. Opioid pharmacovigilance: A clinical-social history of the changes in opioid prescribing for patients with co-occurring chronic non-cancer pain and substance use. Soc Sci Med 2017;186: 87-95.

9. Green CR, Anderson KO, Baker TA, et al. The unequal burden of pain: Confronting racial and ethnic disparities in pain. Pain Med 2003;4:277-294.

10. Dowell D, Haegerich TM, Chou R. CDC Guideline for prescribing opioids for chronic pain-United States, 2016. MMWR Recomm Rep 2016;65:1-49.

11. Chou R, Fanciullo GJ, Fine PG, et al. Clinical guidelines for the use of chronic opioid therapy in chronic noncancer pain. J Pain 2009;10:113-130.

12. Chou R, Huffman LH. Nonpharmacologic therapies for acute and chronic low back pain: A review of the evidence for an American Pain Society/American College of Physicians clinical practice guideline. Ann Intern Med 2007;147: 492-504.

13. Qaseem A, Wilt T, McLean R, Forciea M. Noninvasive treatments for acute, subacute, and chronic low back pain: A clinical practice guideline from the American College of Physicians. Ann Intern Med 2018;166:514-530.

14. Visconti AJ, Santos GM, Lemos NP, et al. Opioid overdose deaths in the city and county of San Francisco: Prevalence, distribution, and disparities. J Urban Health 2015;92:758772.

15. Hurstak E, Rowe C, Turner C, et al. Using medical examiner case narratives to improve opioid overdose surveillance. Int J Drug Policy 2018;54:35-42.

16. Berwick DM. Developing and testing changes in delivery of care. Ann Intern Med 1998;128:651-656. 
17. Kamper SJ, Apeldoorn AT, Chiarotto A, et al. Multidisciplinary biopsychosocial rehabilitation for chronic low back pain: Cochrane systematic review and meta-analysis. BMJ 2015;350:h444.

18. Coates J, Gething F, Johnson MI. Shared medical appointments for managing pain in primary care settings? Pain Manag 2017;7:223-227.

19. Gardiner P, Dresner D, Barnett KG, et al. Medical group visits: A feasibility study to manage patients with chronic pain in an underserved urban clinic. Glob Adv Health Med 2014;3:20-26.

20. Kabat-Zinn J, Lipworth L, Burney R. The clinical use of mindfulness meditation for the self-regulation of chronic pain. J Behav Med 1985;8:163-190.

21. Plews-Ogan M, Owens JE, Goodman M, et al. A pilot study evaluating mindfulness-based stress reduction and massage for the management of chronic pain. J Gen Intern Med 2005;20:1136-1138.

22. Orme-Johnson DW, Schneider RH, Son YD, et al. Neuroimaging of meditation's effect on brain reactivity to pain. Neuroreport 2006;17:1359-1363.

23. Linden A, Butterworth SW, Prochaska JO. Motivational interviewing-based health coaching as a chronic care intervention. J Eval Clin Pract 2010;16:166-174.

24. Kivela K, Elo S, Kyngas H, Kaariainen M. The effects of health coaching on adult patients with chronic diseases: A systematic review. Patient Educ Couns 2014;97:147157.
25. Maslach C, Jackson S, Leiter M. The Maslach Burnout Inventory Manual. Palo Alto, CA: Consulting Psychologists Press, 1996.

26. Dolan ED, Mohr D, Lempa M, et al. Using a single item to measure burnout in primary care staff: A psychometric evaluation. J Gen Intern Med 2014;30:582-587.

27. Dresner D, Gergen Barnett K, Resnick K, et al. Listening to their words: A qualitative analysis of Integrative Medicine Group visits in an urban underserved medical setting. Pain Med 2016. pii: pnw030. [Epub ahead of print].

28. Gardiner P, Lestoquoy AS, Gergen-Barnett K, et al. Design of the integrative medical group visits randomized control trial for underserved patients with chronic pain and depression. Contemp Clin Trials 2017;54:25-35.

29. Hurstak E, Kushel M. Pain Care on a New Track: Complementary Therapies in the Safety Net. Oakland, CA: California Healthcare Foundation, 2016:1-27.

Address correspondence to: Emily E. Hurstak, MD, MPH, MAS Division of General Internal Medicine Zuckerberg San Francisco General Hospital University of California, San Francisco 1001 Potrero Avenue, 1 M3 San Francisco, CA 94110

E-mail: emily.hurstak@ucsf.edu 